
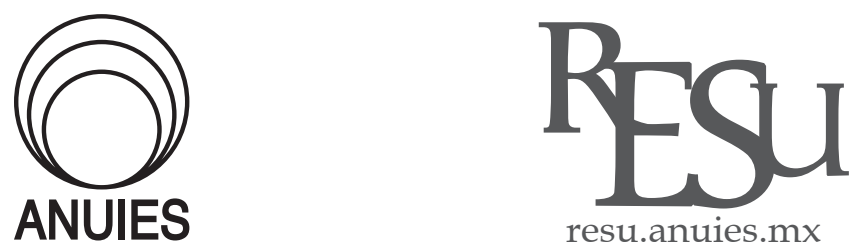

ARTí́CULO

\title{
Cultura digital y cambio institucional de las universidades
}

\author{
Digital culture and institutional change in universities
}

\author{
Miguel Ángel Casillas Alvarado y Alberto Ramírez Martinell* \\ *Centro de Investigación e Innovación en Educación Superior de la Universidad Veracruzana, Xalapa, Veracruz, \\ México. Correo electrónico: mcasillas@uv.mx y albramirez@uv.mx
}

Recibido el 11 de octubre del 2018; aceptado el 04 de julio del 2019

\section{Resumen}

Con la llegada y el posterior arraigo de una cultura digital en las instituciones de educación superior, las comunidades académicas se modificaron. Sus rutinas, formas de gestión, maneras de interactuar y de relacionarse con otros miembros de la comunidad, de investigar, de dar y de tomar clases o de acceder a la información en todos los casos, se vieron permeados por lo digital. Pero esto no sólo se da de forma superficial en la universidad, como se suele dar en otros niveles educativos; la profundidad con la que llega a los campos disciplinarios, que es distinta pero notoria en cada caso, es elemental para integrar las Tecnologías de la Información y de la Comunicación en los planes y programas universitarios.

De acuerdo con los resultados de investigación, la cultura de lo digital en áreas de alta especialización procuradas en la Universidad se refleja en los dispositivos que utilizan los universitarios, los programas informáticos para la manipulación de datos y simulación o las revistas digitales y bases de datos de prestigio y de alta valoración, además de en las formas en que la comunidad académica de una disciplina dada interactúa con estos artefactos digitales, los valora y pone en juego para mejorar sus prácticas disciplinarias y atender una serie de necesidades propias de su campo.

Palabras clave: Educación Superior; Tecnologías de la Información y de la Comunicación; Cultura Digital; Cambio Institucional; Incorporación de TIC 


\begin{abstract}
With the arrival and the popularization of a digital culture in Higher Education Institutions, the academic communities changed. Their routines, managerial procedures, ways of interacting and relating to other members of the community as well as the forms of engagement in research activities, teaching and information access have been influenced by a digital matter. But this is not only granted in the surface of the university activities as it happens in other educational levels. It arrived with diverse intricacies to the disciplinary fields, different in each case, but still notorious, as it takes part of the Information and Communication Technologies to the University Curriculum.

According to research results in the University high specialization areas, the digital culture can be observed in the kind of devices, software or data manipulation techniques they use, the type of journals they read and the ways the academic community of a given disciplinary field interact with the digital items, use and consider them for improving their practices and solving accurate needs of a specific academic field.
\end{abstract}

Keywords: Higher Education; Information and Communication Technologies; Digital Culture; Institutional Change; ICT Incorporation

\title{
Introducción
}

$\mathrm{L}$ a revolución tecnológica y la cultura digital determinan cambios importantes en las organizaciones, incluidas las instituciones educativas. Las funciones sustantivas de los académicos universitarios han sufrido cambios importantes. En la docencia, las herramientas para la presentación de contenidos, las plataformas de aprendizaje distribuido y los motores de búsqueda, así como los repositorios de recursos educativos, son hoy en día recursos comunes para los profesores de prácticamente todos los niveles. Las prácticas de producción y distribución de conocimiento también se modificaron a través del uso de software especializado, del acceso digital a revistas especializadas, bases de datos y bibliotecas virtuales y de redes sociales y comunidades virtuales en las que se pueden enlazar académicos, temas y productos de investigación. La comunicación y la vinculación actual suceden en redes sociales, mensajeros instantáneos, blogs, wikis y otros espacios virtuales que potencian la procuración de una identidad digital de los académicos. Finalmente, la gestión también ha sido modificada por las Tecnologías de la Información y de la Comunicación (TIC). Las tareas de administración, de cálculo y de logística han mejorado gracias a programas informáticos que las automatizan. La llegada de las TIC a la Universidad Veracruzana ha sido analizada en textos introductorios a los 
libros Háblame de TIC (Casillas y Ramírez, 2014; Casillas y Ramírez 2015; Casillas y Ramírez 2016) que abordan el desarrollo de la cultura digital y su impacto en la educación.

La época contemporánea se distingue por el incesante desarrollo de la revolución tecnológica - fundada en la digitalización y el uso extendido de las computadoras y el acceso a Internet - que resulta en la conformación de una cultura digital como nuevo referente simbólico.

El uso cotidiano y la popularización de las TIC en actividades y oficios de todo tipo ha derivado en la construcción de un habitus digital asociado a un sistema de esquemas lógicos o de estructuras cognitivas que organizan las visiones del mundo (o eidos); un conjunto de disposiciones morales (o ethos); de gustos y disposiciones estéticas (o aisthesis); y de posturas, gestos y maneras de ser del cuerpo (o hexis) influenciados por lo digital (Casillas y Ramírez, 2018: 321).

Una de las transformaciones sociales más distintivas que ha ocurrido a partir de la cultura digital es la relativa al cambio del sentido de la realidad y de lo virtual. Con este tipo de cultura, la realidad se amplió y se enriqueció con la propia de lo virtual. Ahora la realidad de lo físico se complementa de notificaciones del Smartphone y con mensajes y signos de redes sociales, se puede capturar mediante una cámara fotográfica de calidad, cada día mejorada y enriquecida por filtros y ediciones sencillas pero visualmente poderosas y digitalmente socializadas. Ahora, a la realidad de lo físico, de lo tangible, de lo presente, la acompañan nuevas realidades: la virtual, la aumentada y la mixta. No la sustituyen, al contrario, sirven para crear nuevas dimensiones, nuevas identidades sociales, nuevos mundos a los que tenemos acceso a través de equipos digitales con acceso a Internet.

En las universidades está ocurriendo una silenciosa transformación, la base tecnológica de la investigación, de la docencia y de la difusión de la cultura está siendo transformada por el uso masivo de computadoras, mediante imágenes visuales que se proyectan en una infinidad de pantallas y, específicamente, en un cambio radical de instrumentos y de dispositivos físicos, en software especializado, en páginas y bases de información, y en términos de los recursos educativos abiertos. Hablamos de una explosión de software especializado que distingue a cada campo o disciplina de estudios. Pareciera una obviedad, pero en realidad las universidades no lo han visto, no lo han reconocido, ni diseñado estrategias o políticas institucionales específicas: siguen empecinadas en una visión general, homogeneizante, con políticas únicas y de alcance universal. Nuestras investigaciones nos han conducido a explorar este fenómeno, a enfatizar la perspectiva de las disciplinas en el desarrollo tecnológico, a reconocer cómo cada campo profesional está uti- 
lizando software específico, a observar que cada campo disciplinario tiene determinados aparatos y dispositivos, y consulta muy particulares bases de datos, revistas, blogs y fuentes de información (Ramírez y Casillas, 2015b); véanse también los reportes sobre los talleres para incorporar las TIC al curriculum disponibles en el blog brecha digital: https://www.uv.mx/blogs/ brechadigital/reportes_sd/.

La base científica del artículo se encuentra en investigaciones sobre la historia de la incorporación de las Tic a las universidades (Casillas y Ramírez, 2015; Casillas, Ramírez, Carvajal, y Valencia, 2016) sobre la incorporación de las TIC al curriculum universitario (Ramírez y Casillas 2016; Ramírez y Casillas, 2014; Ramírez y Casillas, 2015a y Ramírez y Casillas, 2017); y sobre el cambio en las profesiones y disciplinas que hemos documentado (Casillas, Ramírez, Luna y Marini, 2017; Morales y Ramírez, 2015; Martínez, Morales y Ramírez, 2015). Sobre la base de todas estas indagaciones, el problema del cambio institucional y sus efectos organizacionales se nos revela como el principal obstáculo para la modernización universitaria.

Aquí se propone que la incorporación de las TIC en las instituciones de educación superior debe darse de forma reflexiva, legítima e inclusiva, y es necesaria una planificación institucional de un desarrollo tecnológico con base académica, pues consideramos que su incorporación en los campos disciplinarios debe ser el fundamento de cualquier política y estrategia informada tendientes a superar a la vieja capacitación genérica y a las políticas homogéneas de equipamiento y de fortalecimiento de la infraestructura digital.

\section{La revolución tecnológica}

Al hablar de una Revolución Tecnológica hacemos referencia a los productos, artefactos y sistemas de carácter digital que en torno a las computadoras, redes, Internet, teléfonos inteligentes y demás aplicaciones están transformando el mundo, las formas en que la información fluye y asimismo los esquemas y medios para la comunicación humana. Todos los oficios, y más las prácticas profesionales, están siendo transformados por sendas revoluciones (Castells, 2002):

El punto de partida de las políticas TIC propiamente dichas se ubica en los años noventa y está ligado con la integración de la informática y las telecomunicaciones, lo que produce una revolución cualitativa en todos los campos, incluido el educativo. Las posibilidades de conexión y de acceso a Internet son centrales en el cambio de paradigma que representan estas 
tecnologías, en tanto se abre un nuevo espectro de posibilidades para la enseñanza y el aprendizaje, junto con la posibilidad de democratizar el acceso y la inclusión de vastos sectores sociales de la ciudadanía, que hasta ese momento tenían vedada la oportunidad de hacerlo. Las posibilidades de acceso a la información, a enciclopedias y bibliotecas, e incluso la participación colectiva en trabajos colaborativos, crean un nuevo escenario para el desarrollo de la educación. (Lugo, López, Toranzos, y Corbetta, 2014: 34)

El avance tecnológico en relación con la telefonía móvil y el cómputo ha dado lugar a la creación de tecnologías como laptops, tabletas y teléfonos inteligentes cuyas características principales son la portabilidad, la conectividad y la ubicuidad, que favorecen la búsqueda de información, la hiperconexión y el uso de redes sociales digitales.

A nivel global se observa un incremento en el acceso, la posesión y el uso de dispositivos digitales en prácticamente todos los ámbitos de la vida diaria de las personas, gracias al aumento en la oferta y la diversificación de modelos de los equipos. Millones de personas en todos los países utilizan teléfonos inteligentes, se conectan a Internet y participan intensamente de las redes sociales. También es verdad que hay enormes diferencias en términos de acceso, de cobertura, de apropiación tecnológica y a nivel de uso social de las TIC entre los países del primer mundo y en el resto de las naciones. Al interior de los países también hay enormes diferencias de orden económico, social, geográfico, de acceso y de apropiación. Sin embargo, ubicados al final de la segunda década del siglo y hasta donde es posible avizorar sobre el futuro próximo, la tendencia general apunta hacia la ampliación de los recursos y los servicios de la comunicación y el intercambio de información, así como del uso social de las Tic en todas las esferas de la vida de los seres humanos.

La revolución tecnológica ha creado un nuevo mundo simbólico que comprende los modos de pensamiento individual y grupal que comprenden el conjunto de referentes desde los cuales se mira y se interpretan los usos de las TIC hasta la ampliación del dominio técnico, los usos y prácticas, las actitudes, las representaciones sociales y valores que se desarrollan en torno al ciberespacio y al uso masivo de las computadoras. Hay aproximaciones previas a esta noción en diferentes trabajos: Casillas y Ramírez (2015, con fundamento en Levy, 2007) y Castells (2002).

Específicamente para los jóvenes se da una naturalización del mundo digital:

En el contexto tecnocultural actual, la relación entre el mundo real y el mundo virtual no puede entenderse como un vínculo entre dos mundos independientes y separados, que eventualmente coinciden en un punto, 
sino como una cinta de Moebius, donde no existe un adentro y un fuera, y donde es imposible identificar límites entre ambos. Para las nuevas generaciones, cada vez más, la vida digital se fusiona con su domesticidad como un elemento más de la naturaleza. (Lugo, López, Toranzos, y Corbetta, 2014: 21)

Además de desarrollar un nuevo sentido de multitareas donde simultáneamente los jóvenes desarrollan diversas actividades a la vez, especialmente en el espacio digital, en el tema específico de la cultura digital se podría decir que se está creando una nueva inteligencia colectiva:

[...] las nuevas generaciones residen en ambos mundos a la manera de cyborgs, como una fusión de inteligencia natural y artificial. Este nuevo entorno tecnológico que se fue configurando ofrece a las generaciones jóvenes nuevas posibilidades y modalidades de interacción. El surgimiento de una plataforma tecnológica, que se constituye, a la vez, en entorno abierto, facilita el desarrollo de modos de producir que estimulan la "creación colectiva y colaborativa" y generan un conocimiento compartido que se elabora entre diferentes nodos o sujetos, en intercambios de diferentes intensidad, flujo y magnitud. Se trata de aquello que comienza a conocerse como inteligencia colectiva. (Lugo, López, Toranzos, y Corbetta, 2014: 22)

La nueva cultura digital está generando un cambio en el sentido de la propiedad y la autoría. Se está desarrollando la cultura de acceso abierto, se practica el trabajo colaborativo, se han dejado de acumular objetos culturales digitales para consumirlos en línea o en streaming; se está desarrollando un tipo de lectura hipertextual en las enciclopedias y glosarios de creación colectiva (y permanente) (Lugo, López, Toranzos, y Corbetta, 2014: 23).

Con la cultura digital se ha acelerado el tiempo histórico y se ha redimensionado el mundo. A través de las redes sociales, no sólo el consumo de información es el que impera. Son las ideologías dominantes las que dan lugar a dinámicas sociales estandarizadas a nivel global habilitando a los jóvenes para que organicen el mundo de nuevas formas:

Velocidad, aceleración, instante, capacidad de procesamiento, duplicación del conocimiento, todas ellas son claves que deben ser consideradas a la hora de comprender las subjetividades de época.

Los jóvenes estudiantes del presente están entrenados cada vez más en categorías de la experiencia (espacio, tiempo y velocidad) que los adultos no siempre comparten. En consecuencia, asistimos a nuevas formas de organizar y construir el mundo: multilineal, en paralelo, en redes, de nodos frente a centro, desde periferias con jerarquías plurales y, sobre todo, en una secuenciación discursiva arborescente. (Lugo, López, Toranzos, y Corbetta, 2014: 29) 
En la actualidad la información es ampliamente accesible y genera nuevas posibilidades de apropiación social del conocimiento. Por ejemplo, los sistemas de información online están cambiando el sentido tradicional del periodismo y la comunicación, las bibliotecas digitales favorecen el acceso a libros y materiales bibliográficos que en el pasado eran prácticamente inaccesibles para el gran público, los museos y galerías han abierto sus puertas y colecciones. Hay una tendencia al libre acceso de bienes culturales que antes eran asequibles y exclusivos para unos cuantos.

El trabajo se ha transformado. Todas las ramas y todos los procesos económicos han incorporado las computadoras e Internet en sus prácticas laborales. Todos los oficios y profesiones están utilizando nuevos dispositivos digitales, fuentes de información, programas informáticos y aplicaciones cada vez más específicos, con mayor potencia de interconexión aunque con una gran dependencia a la conectividad.

En el marco de la cultura digital se transforman los procesos y dinámicas de la comunicación humana, se modifican las prácticas e interacciones sociales cotidianas cuando se diversifican las formas de encuentro e interacción. El correo postal para el intercambio de cartas y el telégrafo prácticamente han desaparecido; incluso el viejo teléfono de conexión fija ha sido desplazado por los sistemas de mensajería instantánea, por las videollamadas, por el intercambio de mensajes a través de las redes sociales. En las prácticas cotidianas escolares son cada vez más frecuentes los grupos de Facebook o de mensajeros instantáneos instalados en móviles como Whatsapp o Messenger, que amplifican las posibilidades de interacción entre estudiantes y profesores.

En términos del gobierno y de la gestión, la cultura digital está favoreciendo la rendición de cuentas, el acceso y la transparencia, no sólo porque la información es pública y se difunde mejor, sino porque hay un mayor control social de los actos del gobierno. En efecto, se está desarrollando una nueva ciudadanía, mucho más crítica, más informada y vigilante de los planes y acciones gubernamentales. Con las redes sociales y los sitios web especializados se ha ampliado la esfera pública, la participación ciudadana y el infoactivismo.

El cambio tecnológico ha impulsado la educación continua y la necesidad de reaprender en todos los ámbitos de la producción, debido a la disminución en los costos de equipos de cómputo y el énfasis en las habilidades para resolver problemas. Hay un incremento en empleos que demandan mayores habilidades cognitivas por encima de las manuales, lo que motiva que las personas tengan que volver a la escuela (Casillas, et al., 2003; Goos y Manning, 2007; Casillas y Dorn, 2013, citados en Frey y Osborne, 2013). También hay reasignaciones de puestos de trabajo, donde empleados de mandos me- 
dios han sido cambiados a áreas operativas en virtud de que sus labores requieren esfuerzo físico y son por ende menos susceptibles a la informatización (Casillas y Dorn 2013 citado en Frey y Osborne, 2013).

Casillas, Ramírez, Luna, y Marini (2017) manifiestan que la expansión de empleos con habilidades superiores se puede explicar porque disminuye el precio de las tareas rutinarias, que gracias a las computadoras, el trabajo se centra en servicios creativos. El progreso tecnológico ha producido cambios en la composición de ocupaciones y oficios, incluidos los campos de agricultura, artesanías, manufactura, servicios y labores directivas (Brynjolfsson y McAfee, 2011, citado en Frey y Osborne, 2013). Con el actual avance tecnológico, los algoritmos para el procesamiento de grandes volúmenes de datos permiten el reconocimiento de patrones, lo que posibilita que una computadora pueda efectuar fácilmente el trabajo de una amplia gama de tareas no rutinarias que requieran de un esfuerzo cognitivo para su resolución (Brynjolfsson y McAfee, 2011; MGI, 2013, citados en Frey y Osborne, 2013). Además, robots avanzados están generando sentidos y destrezas para efectuar un mayor número de tareas manuales (ifr, 2012b; Robótica-VO, 2013; mgi, 2013, citados en Frey y Osborne, 2013), por lo cual se prevé que la naturaleza del trabajo cambiará en todos los campos y profesiones:

Esta evidente transformación en el oficio, en las prácticas profesionales y en la vida social misma debe ser asumida reflexivamente por los académicos expertos de cada campo profesional y reflejada en la formación de los estudiantes universitarios. Es imperiosa una discusión que conduzca a la modernización de los planes y programas de estudio de todas las carreras y niveles educativos, que precise cuáles son los saberes digitales que distinguen a cada campo disciplinario y a sus especialidades, y que formalice el compromiso con una educación moderna y contemporánea. (Casillas, Ramírez, Luna, y Marini, 2017: 48)

\section{La cultura digital y la educación}

La transformación cultural que representa la ampliación en el uso social de las computadoras también ha llegado a la educación, y específicamente a la educación superior; podemos observar el desarrollo de nuevas formas de lectura, de escritura, de enseñanza, de aprendizaje, de producción y de difusión del conocimiento. El amplio desarrollo de recursos educativos está modificando las posibilidades de la enseñanza en todos los niveles y ya hay una clara tendencia hacia una convergencia multimodal. 
La comunicación entre los agentes de la educación se expande y multiplica: estudiantes y docentes dialogan en línea y tienen abiertos las 24 horas canales de comunicación asincrónicos. Los docentes comparten lecturas, discuten y elaboran documentos electrónicos; los estudiantes elaboran tareas y proyectos y los entregan en formato electrónico; los productos son enriquecidos con multimedia y frecuentemente evidencian el aprendizaje y el trabajo colaborativo escolar.

En la educación superior se han transformado la gestión, la administración, la enseñanza, la investigación, la difusión, las formas de comunicación e intercambio de información. Cada vez son más dominantes los sistemas de gestión y de administración, no sólo para lo referido a las cuestiones presupuestales y de gestión del personal, sino para la administración escolar, la planeación académica y la evaluación. La enseñanza se modifica con la utilización de simuladores, dispositivos digitales, programas informáticos y fuentes de información de alta especialización en donde los estudiantes encuentran textos accesibles en formato digital. Los nuevos modos de producción del conocimiento tienen una base tecnológica muy desarrollada; cada vez hay mayor diversidad de equipos, laboratorios, dispositivos y software que se utiliza en la investigación científica.

El mayor dinamismo del cambio tecnológico está pasando por las disciplinas académicas y es el desarrollo del software especializado el epítome de la incorporación de las TIC a las culturas académicas:

En el terreno de la investigación las cosas están cambiando radicalmente. Todos los estudiosos del tema coinciden que vivimos una profunda transformación de los sistemas de producción científica (Gibbons et al., 1994; Gibbons, 1998; Brunner, 2003; Castells, 2006). A través de las TIC son cada día más frecuentes los intercambios, el trabajo deslocalizado y la colaboración en red para favorecer la producción de los conocimientos. La información se comparte ahora en tiempo real y en volúmenes inmensos de datos. El mayor dinamismo del cambio tecnológico que ocurre en las universidades está pasando por las disciplinas académicas y el desarrollo del software especializado: no sólo se ha expandido el uso de computadoras en todos los campos científicos, sino que se están desarrollando aplicaciones específicas en grandes cantidades. Todos los aparatos y todos los procesos se están viendo transformados por la tecnología digital, y en la punta del conocimiento están los universitarios. (Casillas y Ramírez, 2016: 25)

A pesar de su intensidad, y de la velocidad con la que se han dado, los cambios que derivan de la revolución tecnológica en el entorno universitario han ocurrido de manera improvisada, escasamente regulada, sin planes ni metas 
razonables, sin conocimiento de cuánto saben de TIC nuestros profesores y nuestros estudiantes cuando ingresan a la institución y sin precisión sobre su perfil tecnológico de egreso.

Un breve resumen puede sintetizar la experiencia universitaria acumulada en los últimos años. Hasta ahora nos hemos orientado por ocurrencias y buena voluntad, a través de procesos improvisados cuando deberíamos haber trascendido a decisiones informadas, legítimas, reflexivas, transparentes, planeadas y sujetas a evaluación. Dada la heterogeneidad y la falta de un acuerdo institucional, predomina el azar de que ocurra en el curriculum, cuando debería ser la base de la experiencia escolar en cada asignatura. En materia de compras, infraestructura, capacitación, han predominado las políticas generales y homogéneas, cuando debería de haber otras generales y específicas a las necesidades de desarrollo de cada campo disciplinario.

\section{La base académica para la incorporación de las TIC a la ES}

Sólo hasta que las universidades puedan construir un diagnóstico de necesidades tecnológicas para cada campo de estudios y para el pleno cumplimiento de las funciones de docencia, de investigación y de difusión de la cultura, cuando sean capaces de definir un sentido académico para el uso de las TIC, entonces será posible que se construyan políticas e iniciativas institucionales integrales que busquen racionalizar el uso de los recursos económicos y tecnológicos.

De manera desafortunada, en la enorme mayoría de las instituciones de educación superior las decisiones sobre la incorporación y el desarrollo de las TIC han sido un asunto de tecnólogos, ingenieros en sistemas y en cómputo. Muchas de las direcciones de tecnologías están ubicadas organizacionalmente en el sector administrativo, lejos del mundo académico de sus necesidades y de una oportunidad real de incorporar las Tic a la Universidad. Los académicos que pretenden hacer uso de tecnologías - páginas web, dispositivos o software - deben primero pedir permiso a un administrador para que se desbloquee el sistema o se le otorguen privilegios para la operación del aparato tecnológico extraño. El personal administrativo encargado del área de tecnologías de información de la universidad, sin ningún criterio académico, debe valorar si la página web que originalmente estaba bloqueada puede ser visitada en la red institucional o no, o si el software que se quiere instalar en un equipo de cómputo de la universidad es adecuado. 
La política institucional de la prohibición, la desconfianza y el bloqueo debe cambiar. El empleo de las Tic en las universidades debe responder a las necesidades disciplinarias derivadas de una reflexión académica entre los colegios de profesores que son los que deberían, al menos en un sentido enunciativo, administrar sus necesidades tecnológicas y de acceso a la información.

La incorporación de las TIC a las ies debe darse de forma reflexiva, legítima e inclusiva. Reflexiva en el sentido de que tiene que ser racional, basada en el conocimiento de las necesidades institucionales y no en el sentido común o en ocurrencias; planeada y no improvisada; transparente y resultado de licitaciones públicas; considerar el impacto ambiental y los riesgos asociados al uso de las TIC. Legítima porque no puede ser una imposición o una decisión arbitraria, por más eruditos que puedan ser los tomadores de decisiones; para que ocurran los cambios institucionales, los académicos tienen que participar activamente en la definición del rumbo; las decisiones deben tener una base colegiada en la que se garantice una amplia participación; los avances de los acuerdos deban ser revisados periódicamente por parte de la comunidad académica. Inclusiva para poder incorporar a todos, para ampliar las capacidades tecnológicas de todos los universitarios y generar condiciones de equidad para el acceso y la apropiación tecnológica.

Las instituciones no pueden seguir improvisando, es necesario un plan institucional de desarrollo tecnológico, construido en primer lugar con base en las necesidades de las disciplinas académicas, que trascienda la capacitación genérica y las políticas generales y homogéneas de equipamiento y de procuración de la infraestructura digital. Es necesario un plan que defina una postura y una filosofía institucionales sobre las TIC, con objetivos a corto, mediano y largo plazo; que estructure prioridades, metas y acciones sujetas a la evaluación que favorezcan el aprendizaje institucional.

El plan de desarrollo debería comprender políticas que orienten la incorporación de las TIC en cuatro niveles jerárquicos:

- El proyecto educativo con el que las tic contribuyen a la realización de las funciones sustantivas de las instituciones de educación superior (docencia, investigación y difusión de la cultura).

- El proyecto organizacional que define a la institución: sus formas de gobierno, sus formas de organización y el modo en cómo se desarrollan sus funciones administrativas y que pueden ser mejoradas con el uso de las TIC.

- Los servicios de cómputo entendidos como el conjunto de recursos tecnológicos, de conectividad, de licenciamiento de software y adquisición de equipo, así como su mantenimiento y su soporte técnico.

- La capacitación y la formación continuas de la comunidad universitaria en materia de TIC, en función del proyecto educativo, la naturaleza organizacional y el conjunto de recursos tecnológicos disponibles por parte de la institución. 
Para ser realistas, en el contexto universitario cualquier plan de desarrollo tecnológico debe estar basado en: 1) una perspectiva histórica, 2) debe generar una postura cultural respecto de las TIC, 3) debe tener sustento en la diversidad disciplinaria y 4) debe comprender las diferencias sociales y de dominio tecnológico que caracterizan a los universitarios.

La primera se trata de una perspectiva de orden histórico-social en la que la comunidad universitaria es sensible a la evolución histórica de las TIC en su entorno social y en su institución, y que a su vez sea capaz de reconocer que la incorporación de las Tic es un fenómeno reciente, que se encuentra en construcción y que en el corto plazo ya esboza una dirección. Se trata de una historia que debemos documentar pues se están transformando de manera radical los procesos y funciones universitarias (cambios en los modos de enseñar, de producir y difundir el conocimiento), se están reestructurando los espacios universitarios convencionales (como las aulas, los laboratorios, las bibliotecas), y se está reconfigurando la noción del espacio con la conformación de nuevos espacios abiertos (Wifi) y virtuales.

La segunda es una perspectiva que reconoce que la incorporación de las TIC a la educación no es algo natural ni que llegará por si sola, sino que es necesaria una reflexión institucional sobre el conjunto de referentes, técnicas, prácticas, actitudes, modos de pensamiento, representaciones sociales y valores que se desarrollan en torno al ciberespacio y al uso masivo de las computadoras. Es necesario que la institución asuma una postura, defina una filosofía, oriente sus decisiones tecnológicas desde la complejidad académica, ética y ambiental.

En cuanto al tercer punto, a pesar de las políticas institucionales en las que se ve a la integración de las TIC como un proceso homogéneo, la realidad es que dada la naturaleza del trabajo académico (Clark, 1987) y la heterogeneidad institucional que estructura y delimita el desarrollo de las disciplinas, la incorporación de las TIC a las disciplinas académicas sucede de manera diferenciada.

Finalmente, también es necesaria una perspectiva de orden sociológico en la que se consideren las Tic como un capital tecnológico (Casillas, Ramírez y Ortiz, 2014), que en el campo educativo está distribuido de manera desigual y que es necesario distribuir con equidad entre los agentes universitarios. Por tanto las universidades deben tener información precisa sobre el grado de apropiación tecnológica, que mide el dominio de los saberes digitales. El uso de las TIC en la educación valoriza un nuevo tipo de saberes y exige un nuevo tipo de habilidades y destrezas que no han sido plenamente reconocidas pues no hay un diagnóstico que permita saber qué disposiciones poseen estudiantes y profesores. Para la Universidad es necesaria la interpretación 
de corte sociológico de las diferencias que distinguen a los estudiantes y profesores por su grado de apropiación tecnológica, por su grado de afinidad y proclividad hacia las tecnologías, y por el grado de acceso y disponibilidad que tienen.

\section{Conclusiones}

El cambio institucional que deriva de la incorporación de las TIC es ineludible y no admite retraso. La discusión no debe ser entre si se incorporan o no las TIC a las actividades sustantivas de los universitarios, sino sobre la profundidad y el impacto de su incorporación. En algunos casos esta discusión es al nivel de productos o versiones de programas informáticos por ser utilizados; en otros es sobre las asignaturas en las que el software puede intervenir; y en otros más puede ser sobre estrategias de enseñanza y uso del software y la información que se genera al manipularlo. Pero en todos los casos se trata de discusiones de alto nivel de reflexión, legitimidad e inclusión, en las que los académicos se apropian de las TIC y las ven como instrumentos disciplinarios, tal y como lo hicieron cuando este tipo de herramientas en el orden de lo físico representaban epítomes de la disciplina, sea el teodolito para los ingenieros topógrafos y geodestas, el estetoscopio para el médico, la Constitución para los abogados, la regla $\mathrm{T}$ y el restirador para los arquitectos y diseñadores gráficos.

Una nueva brecha diferencia a los países, a las regiones, a las instituciones, a los individuos y grupos sociales: la que se distingue por el acceso y el dominio de los recursos tecnológicos; por la incorporación de las TIC a las dinámicas laborales, de estudio, de participación ciudadana.

Aquí hemos argumentado a favor de una perspectiva que considera que el cambio necesario debe ser reflexivo, legítimo e inclusivo. En contra de las tendencias a la improvisación, aquí sostenemos la necesidad de un plan de desarrollo tecnológico, que debe orientar el cambio para definir prioridades, metas y acciones, y para que sea evaluable.

Las TIC son muy importantes en el medio universitario como para dejarlas en manos de tecnólogos, y dada la naturaleza específica del trabajo que ahí se desarrolla, el plan de desarrollo tecnológico debe tener una base académica. Para ser realista, el plan de desarrollo tecnológico debe estar basado en una perspectiva histórica, generar una postura cultural respecto de las TIC, tener sustento en la diversidad disciplinaria y comprender las diferencias sociales y de dominio tecnológico que caracterizan a los universitarios. 


\section{Referencias}

Casillas, Miguel Ángel y Ramírez-Martinell, Alberto (Coords.). (2015a). Génesis de las tic en la Universidad Veracruzana: Ensayo de periodización. México: Productora de Contenidos Culturales Sagahón Repoll.

Casillas, Miguel Ángel y Ramírez-Martinell, Alberto (Coords.). (2015b). Háblame de тIC 2: Internet en Educación Superior. Córdoba, Argentina: Editorial Brujas.

Casillas, Miguel Ángel y Ramírez, Alberto (Coords.). (2016). Háblame de TIC 3: Educación Virtual y Recursos Educativos. Córdoba, Argentina: Brujas.

Casillas, Miguel Ángel y Ramírez, Alberto (2018). El habitus digital: una propuesta para su observación. En Roberto Castro y Hugo José Suárez (Coords.), Pierre Bourdieu en la sociología latinoamericana: el uso de campo y habitus en la investigación (pp. 317-341). Cuernavaca, Morelos: Universidad Nacional Autónoma de México, Centro Regional de Investigaciones Multidisciplinarias.

Casillas, Miguel Ángel Ramírez-Martinell, Alberto Carvajal, M., y Valencia, K. (2016). La integración de México a la sociedad de la Información. En E. Téllez (Coord.), Derecho y тіс. Vertientes Actuales (pp. 1-31). México: Universidad Nacional Autónoma de México, Instituto de Investigaciones Jurídicas.

Casillas, Miguel Ángel, Ramírez-Martinel, Alberto , Luna, Marisol y Marini, Verónica (2017). Ensayo de definición del perfil tecnológico del abogado. En Evelyn Téllez, Alberto Ramírez, y Miguel Casillas. (Coords.). El abogado actual frente al derecho informático y su enseñanza (42-60). Xalapa-Veracruz: Biblioteca Digital de Humanidades, INFOTEC.

Castells, Manuel (2002). La Era de la Información. Vol. I: La Sociedad Red. México. Distrito Federal: Siglo XXI Editores.

Clark, Burton (1987). The Academic Profession. National, Disciplinary, and Institucional Settings. Estados Unidos de América: Universidad de California.

Frey, Carl Benedikt \& Osborne Michael A (Septiembre, 2013). The future of employment: how susceptible are jobs to computerisation? Oxford Martin Programme on Technology and Employment, University of Oxford. Recuperado de http:/ / www.oxfordmartin.ox.ac.uk/publications/view/1314

Lévy, Pierre (2007). Cibercultura: Informe al Consejo de Europa. España: Anthropos y Universidad Autónoma Metropolitana.

López, Nestor; Lugo, María Teresa; Toranzos, Lilia y Corbetta, Silvina(2014). Informe sobre Tendencias Sociales y Educativas en América Latina 2014. Políticas TIC en los Sistemas Educativos de América Latina. UNESCO, IIPE - UNESCO y Organización de Estados Iberoamericanos para la Educación, la Ciencia y la Cultura. Recuperado de: http://www.siteal.iipe.unesco.org/ sites/default/files/siteal_informe_2014_politicas_tic.pdf

Martínez, Karla Paola; Morales, Ana Teresa y Ramírez Martinell, Alberto (2015). Cultura libre y de acceso abierto de los profesores universitarios: una mirada desde las disciplinas académicas. Revista Virtualis, 6(12), 110-128. 
Morales, Ana Teresa y Ramírez Martinell, Alberto (2015). Brecha digital de acceso entre profesores universitarios, de acuerdo a su disciplina. Revista Debate Universitario, 3(6), 149- 158.

Ramírez-Martinell, Alberto y Casillas, Miguel Ángel (Coords.). (2014). Háblame de тіс: Tecnología Digital en la Educación Superior. Córdoba, Argentina: Editorial Brujas.

Ramírez, Alberto, y Casillas, Miguel (2015). Los saberes digitales de los universitarios. En Jordy Micheli (Coord.), Educación Virtual y Universidad, un modelo de evaluación (pp. 77-106). México: Universidad Autónoma Metropolitana 
